\title{
Caracterizando a campanha presidencial brasileira em 2018 usando dados do Twitter
}

\author{
Emanuelle Azevedo Martins ${ }^{1}$, Kênia Carolina Gonçalves ${ }^{1}$ e Renato Miranda Filho ${ }^{1}$ \\ ${ }^{1}$ Instituto Federal de Educação, Ciência e Tecnologia de Minas Gerais (IFMG) \\ 34.564-070 - Sabará - MG - Brasil \\ emanuelle.azevedo07@gmail.com, \\ \{kenia.carolina, renato.miranda\}@ifmg.edu.br
}

\begin{abstract}
Online social networks are relevant even in elections processes. In this context, this work characterized data collected from Twitter during the Brazilian presidential elections in 2018. For that, the data were analyzed over time as the percentage of followers, the number of mentions to the opposing candidates, the most used words and the tweets shared, liked and retweet. It was possible to identify that the candidate who won the elections was not the candidate with the largest number of followers, but the one that was most cited by his opponents and in the trending topics, which showed a greater variation in his number of followers and accumulated a larger number of likes and sharing of his publications.
\end{abstract}

Resumo. As redes sociais online são relevantes até mesmo em processos eleitorais. Neste contexto, este trabalho caracterizou dados coletados do Twitter durante as eleições presidenciais brasileiras em 2018. Para tanto, foram analisados os dados ao longo do tempo como o percentual de seguidores, a quantidade de menções aos candidatos oponentes, as palavras mais utilizadas e os tweets compartilhados, curtidos e retweetados. Foi possivel identificar que o candidato que venceu as eleições não era o candidato com o maior número de seguidores, e sim aquele que foi mais citado pelos seus oponentes e nos trending topics, que apresentou maior variação em seu número de seguidores e acumulou maior quantidade de curtidas e compartilhamentos em suas publicações.

\section{Introdução}

A Web 2.0 proporcionou um aumento considerável do uso das redes sociais, da troca de mensagens instantâneas entre usuários e do dinamismo nas interações online. Em 2018 um estudo mostrou que $66 \%$ da população brasileira estava conectada à Internet, ou seja, 139.1 milhões de pessoas, e que $62 \%$ dos brasileiros fazem o uso de alguma rede social [Martins 2018]. O impacto das mídias sociais é tamanho que as redes sociais são capazes de afetar inclusive o cenário político de um país. Nas eleições presidenciais no Brasil de 2014, candidatos com menor tempo de propaganda eleitoral na televisão passaram a investir na divulgação online de suas campanhas ${ }^{1}$. Nas eleições presidenciais dos Estados Unidos de 2016, a rede social Facebook teve papel fundamental na campanha do

\footnotetext{
${ }^{1}$ The Economist. (2014). Winning hearts and likes. Disponível em: https://goo.gl/raoqkp . Acesso em: 30 de Janeiro 2019.
} 
presidente Donald Trump, impactando diretamente no seu crescimento de popularidade [Cellan-Jones 2016]. Governos também passaram a utilizar redes sociais para postar comunicados oficiais de forma mais acessível e informal. Neste cenário, uma destas redes sociais com grande relevância é o Twitter, amplamente utilizado por políticos.

No Brasil, as eleições presidenciais ocorrem a cada quatro anos, têm voto secreto e podem acontecer em 1 ou 2 turnos. O voto é obrigatório aos cidadãos brasileiros com idade entre 18 e 70 anos e facultativo para jovens com idade entre 16 e 17 anos, para pessoas com mais de 70 anos e para analfabetos. Um candidato é eleito no primeiro turno obtendo maioria absoluta dos votos válidos. Caso contrário, para eleições presidenciais, os dois candidatos mais bem votados pela população disputam o segundo turno. É eleito o candidato com mais de $50 \%+1$ dos votos válidos ${ }^{2}$. A eleição de 2018 apresentou 13 candidatos à presidência do Brasil, maior número em 29 anos $^{3}$. Esta foi uma eleição marcada pelo uso das redes sociais, da Internet e de fake news como propulsoras das candidaturas por grande parte dos candidatos.

Alguns trabalhos e pesquisas vêm tentando caracterizar o uso e as informações nas redes sociais no cenário político. Em [Attux 2017, Miranda Filho et al. 2014], os autores tentam prever o resultado das eleições. Já em [Camargo et al. 2016], os dados do Tribunal Superior Eleitoral (TSE) foram utilizados para determinar padrões de candidatos a vereadores. Em [Junior et al. 2016], foram analisadas as reações dos usuários ao lerem publicações relacionadas à política em seus perfis nas redes sociais Twitter e Facebook. Em [Vegas et al. 2016], dados do Twitter foram utilizados para caracterizar os perfis de usuários de cada candidato à presidência e os impactos presenciados na rede social durante toda a campanha.

Neste trabalho, o principal objetivo é caracterizar as eleições presidenciais brasileiras de 2018 no Twitter. Com essa caracterização identificamos os termos mais publicados pelos candidatos, a relação entre o volume de seguidores e suas publicações, além de verificar se os candidatos citam com frequência os oponentes. Também avaliamos qual candidato esteve em maior evidência na rede social e se acontecimentos online se relacionam com fatos fora das redes sociais.

Os resultados indicam que o candidato que venceu as eleições foi aquele mais citado nos trending topics ao longo da campanha eleitoral, o mais citado por seus oponentes e que apresentou maior variação nos seus números de seguidores no Twitter. Observamos também que alguns candidatos repetem com maior frequência em suas publicações os próprios slogans e outros possuem também publicações voltadas para o engajamento de seus seguidores. Além disso, foi possível relacionar algumas movimentações no número de seguidores com acontecimentos externos como debates e o atentado ao candidato Jair Bolsonaro [G1 2018].

Este trabalho está organizado da seguinte maneira: a Seção 2 apresenta os trabalhos que serviram de base para este artigo. A Seção 3 descreve a metodologia empregada. A Seção 4 mostra os resultados obtidos e a Seção 5 apresenta as conclusões e propostas

\footnotetext{
${ }^{2}$ Tribunal Superior Eleitoral. (2014). Como são contabilizados os votos nas eleições brasileiras. Disponível em: https://goo.gl/2Rrw3s . Acesso em: 05 de Janeiro 2019.

${ }^{3}$ Poder 360. (2018). Eleições terão 13 candidatos ao planalto, maior número desde 1989. Disponível em: https://goo.gl/FSL1qv . Acesso em: 25 de Fevereiro 2019.
} 
de continuidade deste trabalho.

\section{Trabalhos Relacionados}

Diversos trabalhos de pesquisa avaliam e caracterizam como as pessoas utilizam as redes sociais [Recuero and Zago 2016, Attux 2017, Camargo et al. 2016, Junior et al. 2016, Vegas et al. 2016, Li et al. 2018]. No contexto social de eleições presidenciais, o Twitter já foi avaliado e caracterizado algumas vezes. Alguns trabalhos mostram o impacto que notícias políticas ou sobre candidatos podem causar aos usuários. As mídias sociais são as principais escolhas para a divulgação dessas notícias políticas em campanhas, devido à grande quantidade de pessoas que terão acesso a essas informações e a rapidez de disseminação do conteúdo. O Twitter é uma destas redes sociais com relevância na Internet.

O trabalho [Fourney et al. 2017] retrata notícias falsas vinculadas à campanha eleitoral presidencial de 2016 nos Estados Unidos nos meses anteriores às eleições. Foram feitas análises sobre o tipo de notícias em relação ao tempo de circulação e sobre os padrões de voto dos usuários de sites de notícias falsas. Os autores também mostram as limitações do trabalho por avaliarem apenas dois navegadores e a dificuldade encontrada em definir e verificar notícias falsas. Ao contrário desse trabalho, o presente artigo busca caracterizar as eleições presidenciais no ano de 2018 utilizando dados da rede social Twitter no aspecto das características dos candidatos à presidência.

Em [Junior et al. 2016], os autores fazem o uso das redes sociais Twitter e Facebook para analisar a reação dos usuários ao lerem publicações sobre política em seus perfis, sejam elas com ideologias contrárias, ofensas a um candidato ao qual o usuário prefere ou até sarcasmo e humor. Para a análise foram extraídos dados do Twitter através de hashtags, também aplicado um questionário para os usuários do Facebook e entrevista presencial com algumas pessoas que responderam o questionário. Com os dados obtidos foram apresentados os perfis mais relevantes e sua distribuição de acordo com sexo, idade e formação. O resultado da pesquisa foi que os usuários das redes sociais pesquisadas são interessados em política e se preocupam com a fonte das informações lidas. Neste trabalho, apenas os conteúdos online são avaliados e de forma temporal sem focar nos seguidores dos candidatos.

Com o intuito de prever o resultado das eleições presidenciais brasileiras de 2014, os autores em [Attux 2017] caracterizaram informações da rede social Twitter. Para isso, foram coletados tweets de termos relacionados aos quatro principais candidatos à presidência no período da campanha eleitoral. Os dados traduzidos foram enviados para o algoritmo que classifica os tweets em positivos, negativos ou neutros. O trabalho obteve alguns resultados bem próximos aos da realidade, porém há uma grande quantidade de pessoas que não possuem acesso à Internet, que influi diretamente no resultado obtido. Como trabalhos futuros o autor propõe o acompanhamento dos dados em tempo real e pelo período anterior e posterior as campanhas, realização da predição para outros cargos políticos e desenvolvimento de um algoritmo classificador próprio para a língua portuguesa. Nesse trabalho, diferentemente de [Attux 2017], não houve a intenção de prever o resultado da campanha. O objetivo foi avaliar os conteúdos em português, os números de seguidores, curtidas e compartilhamentos, além de analisar o conteúdo dos tweets dos candidatos. E como os autores sugeriram, foi feita uma análise temporal do conteúdo, 
antes, durante e após a campanha eleitoral.

Os autores em [Vegas et al. 2016] fazem o uso de dados do Twitter para reconhecer os perfis de usuários que acompanham os candidatos à presidência. Além disso, faz estudo da relação entre os políticos e seus seguidores com a análise de tweets, retweets, publicações favoritas, principais termos de seus tweets, além de obter dados como sexo e localização geográfica. Os autores obtiveram resultados relevantes mostrando que eventos de campanha impactam diretamente no volume de curtidas dos tweets. Além disso, o trabalho apresenta os principais termos usados pelos candidatos e mostra os perfis de apoiadores classificados por gênero e localização, por exemplo. Uma sugestão do autor é estudar tweets do público em geral e não só dos candidatos e a realização de análise de sentimentos. Esse artigo foi a referência principal utilizada nesse trabalho, porém no contexto das eleições presidenciais no Brasil de 2018.

\section{Metodologia}

Com o crescente uso das mídias sociais surgiram novas formas de se obter informações a partir dos dados gerados pelos usuários. Na rede social Twitter, por exemplo, os usuários postam conteúdo em forma de tweets, com limite de 280 caracteres e neles podem conter dados como localização, identificação do usuário, hashtags, imagens, vídeos ou gifs. Outro tipo de publicação presente no Twitter é o retweet, que é o compartilhamento de um tweet publicado por outro usuário da rede social ou pelo próprio usuário que o está compartilhando ${ }^{4}$. O Twitter também apresenta os trending topics que são os assuntos mais comentados na rede social, ou seja, os tópicos com maior discussão naquele momento. Para definição desses termos o Twitter utiliza algoritmos para identificação de tópicos populares, podendo ser ou não personalizados de acordo com a localização e preferências dos usuários ${ }^{5}$.

Para análise da campanha presidencial do Brasil no ano de 2018 as seguintes etapas foram seguidas: (1) Coleta dos Dados (subseção 3.1) e (2) Extração de Informações (subseção 3.2). A seguir, apresenta-se a especificação de cada etapa desenvolvida.

\subsection{Coleta dos Dados}

A base utilizada para realização do trabalho foi coletada no Twitter diariamente após às 20 horas e entre os dias 28 de Agosto e 12 de Novembro de 2018, totalizando 12.095 tweets e 3.367 termos dos trending topics.

Foram coletados dados das contas oficiais de todos os 13 candidatos à presidência da república, definidos no dia 15 de Agosto de 2018 [D’Agostino 2018], prazo final para registro das candidaturas junto à Justiça Eleitoral ${ }^{6}$. Nesse primeiro momento os seguintes nomes estavam definidos como candidatos: Álvaro Dias, Cabo Daciolo, Ciro Gomes, José Maria Eymael, Luiz Inácio Lula da Silva, Geraldo Alckmin, Guilherme Boulos, Henrique Meirelles, Jair Bolsonaro, João Amoêdo, João Goulart Filho, Marina Silva e Vera Lúcia.

\footnotetext{
${ }^{4}$ Twitter. (2006). Sobre diferentes tipos de tweets. Disponível em: https://goo.gl/ttRDwi . Acesso em: 23 de Janeiro 2019.

${ }^{5}$ Twitter. (2006). Perguntas frequentes sobre assuntos do momento no Twitter. Disponível em: https://goo.gl/WbTsdt . Acesso em: 23 de Janeiro 2019.

${ }^{6}$ Tribunal Superior Eleitoral. (2017). Confira as principais datas do calendário eleitoral das eleições gerais de 2018. Disponível em: https://goo.gl/SWmfn6. Acesso em: 30 de Janeiro 2019.
} 
Porém, no dia 11 de Setembro de 2018 o candidato Fernando Haddad foi anunciado no lugar de Luiz Inácio Lula da Silva [Kaniak et al. 2018]. Por esse motivo, somente a partir do dia 12 de Setembro começou-se a coleta dos dados de Fernando Haddad, e devido a relevância de Lula na campanha eleitoral de 2018 a coleta de seus dados foi mantida até o final.

Foram desenvolvidos três crawlers, utilizando a API Tweepy ${ }^{7}$. O primeiro script coletor buscou pelos termos dos trending topics no Brasil. Essa coleta retorna os trending topics do momento. Abaixo seguem exemplos de termos coletados dos Trending Topics relacionados às eleições:

- \#MULHERESCOMBOLSONARO

- MARINA E ALCKMIN

No segundo script coletor foram obtidas informações sobre os usuários candidatos à presidência. Foram coletadas a quantidade de usuários que seguem o candidato, quantidade de pessoas que o candidato segue, quantidade de tweets publicados e a descrição de cada um. A descrição é coletada da seção "Bio" do Twitter e é um texto com até 160 caracteres. Abaixo segue um exemplo de uma instância coletada por este script:

- 31|8|20|16|19|4691|224|1313116|CAPITAO DO EXERCITO BRASILEIRO, DEPUTADO FEDERAL ELEITO PELO RJ E CANDIDATO A PRESIDENCIA DA REPUBLICA COM O NUMERO 17.

O último script realizou a coleta dos dados de cada tweet do dia, a partir do último identificador coletado no dia anterior. Foram coletados os tweets por candidato com as seguintes informações: seu id numérico, o número de retweets e curtidas de cada um, data e hora da publicação e o texto do tweet. Caso o tweet seja um vídeo ou uma imagem, o texto conterá o link do tweet e se for um retweet começará com RT e o nome do usuário do tweet original. Ao final da coleta foi contabilizado 12.095 tweets. Abaixo segue um exemplo deste tipo de coleta:

- 1038112690143731712|07|09|17|12|07|11034|67542|AGRADECO DO FUNDO DO MEU CORACAO A DEUS, MINHA ESPOSA E FILHOS, QUE ESTAO AO MEU LADO, AOS MEDICOS QUE CUIDAM DE MIM E QUE SAO ESSENCIAIS PARA QUE EU PUDESSE CONTINUAR COM VOCES AQUI NA TERRA, E A TODOS PELO APOIO E ORACOES!

Após os crawlers, os dados textuais passam por um pré-processamento. Nesta etapa foram retirados as acentuações, caracteres especiais, termos stop-words, quebras de linha e todo o texto foi passado para maiúsculo.

\subsection{Extração da Informação}

A primeira análise dos dados coletados foi nos termos mais frequentemente usados pelos candidatos. Para esta representação, foi utilizada a técnica de nuvens de palavras. Uma nuvem de palavras é uma representação visual de palavras que ganharam importância (frequência) dentro de um conjunto de palavras [Buchdid and Baranauskas 2012]. Para tanto foi utilizada a linguagem Python com a biblioteca WordCloud.

\footnotetext{
${ }^{7}$ Tweepy. An easy-to-use Python library for accessing the Twitter API. Disponível em: http://www.tweepy.org
} 
Em seguida, foram extraídas informações quantitativas dos dados coletados como: total de tweets por candidato, total de tweets que o candidato compartilhou de outra pessoa, total de curtidas e retweets recebidos nos tweets de cada candidato e número de seguidores dos candidatos. Já para calcular o percentual de incremento do número de seguidores de cada candidato ao longo do tempo seguiu-se a Equação (1).

$$
E_{i}=\frac{\text { count }_{i}-\text { count }_{i-1}}{\text { count }_{i-1}} \times 100(\%)
$$

$\mathrm{Na}$ Equação (1) count $_{i}$ representa a quantidade de seguidores do dia e count $_{i-1}$ descreve a quantidade de seguidores do dia anterior. Esse cálculo foi realizado com dados de seguidores em todo o período da coleta.

Para apresentar o relacionamento existente entre os candidatos foi utilizado o grafo de relacionamentos. Em um grafo de relacionamentos existe uma estrutura de vértices e arestas, e caso haja o relacionamento entre os vértices do grafo haverá uma aresta ligando os dois vértices com um valor numérico que representa o peso desse relacionamento. Para isso, cada vértice representa um candidato e para calcular o peso das arestas foi contabilizado o número de vezes em que um candidato citou em seus tweets o nome de outro candidato. Assim, para calcular a frequência das arestas foi pesquisado dentro da base de dados de tweets de cada candidato os seguintes termos para os candidatos: ALVARO, DACIOLO, CIRO, EYMAEL, ALCKMIN, BOULOS, HADDAD, BOLSONARO, AMOEDO, GOULART, LULA, MARINA, MEIRELLES E VERA LUCIA. A pesquisa retornou a quantidade de vezes em que aparece na base de dados especificada $o$ nome do candidato oponente e também o seu próprio nome. Com esses valores, um grafo foi criado para cada candidato a fim de facilitar a visualização.

\section{Resultados}

Esta seção apresenta os resultados obtidos através da caracterização da base de dados coletada no contexto das eleições presidenciais no Brasil no ano de $2018^{8}$.

\subsection{Palavras mais utilizadas pelos candidatos}

A Figura 1 apresenta as nuvens de palavras dos candidatos durante todo o período de coleta. Pode-se destacar a frequência em que os candidatos publicam seus próprios nomes ou seus números para votação. Como exemplo, nas Figuras 1(b) e 1(f), os termos 'CIROPRESIDENTE' e 'MEIRELLES15', respectivamente, se repetem muitas vezes nas publicações.

Também percebe-se que expressões comumente utilizadas pelos candidatos em seus pronunciamentos aparecem em destaque nas imagens. Exemplificando, na Figura 1(a) 'DEUS', 'ABENÇOE' e 'GLORIA'. Na Figura 1(c) 'LULA', na Figura 1(e) 'HADDAD_FERNANDO' e na Figura 1(f) 'CHAMAOMEIRELLES'.

Nota-se a interação dos candidatos com público divulgando debates e entrevistas como na Figura 1(b) mostra 'CIRONAGLOBO'. Também percebe-se conversas com o público e lives, como nas Figuras 1(d) 'LIVE' e 1(b) 'PERGUNTEAOCIRO12'.

\footnotetext{
${ }^{8}$ Foram apresentados os resultados considerados mais relevantes para o estudo em questão. A análise completa está disponível na pasta compartilhada: https://drive.google.com/drive/ folders/1iDdWgP_Nwh31F2CNxBQahyK6P_zlk6S2 ?usp=sharing .
} 


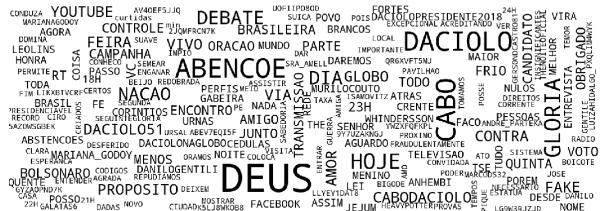

(a) Cabo Daciolo

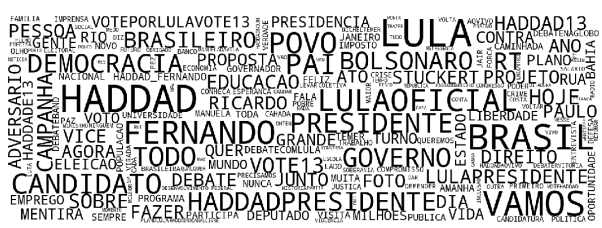

(c) Fernando Haddad

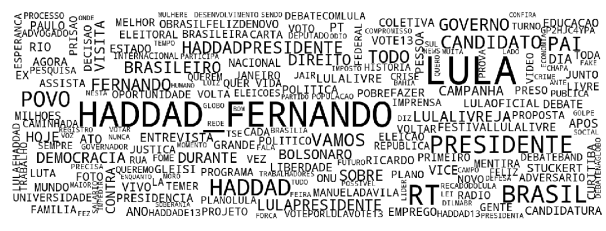

(e) Lula

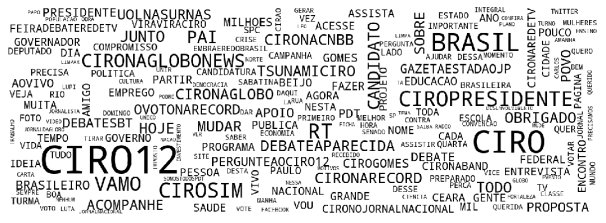

(b) Ciro Gomes

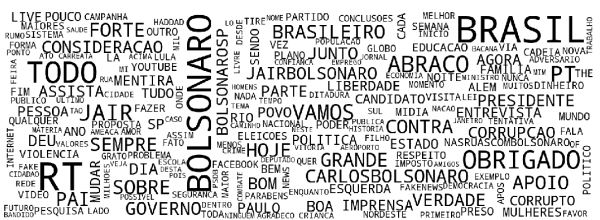

(d) Jair Bolsonaro

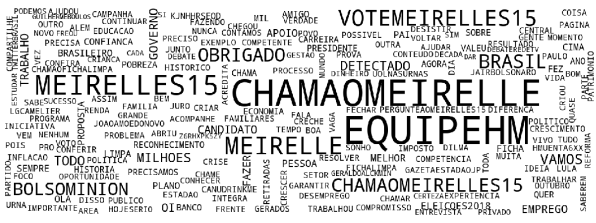

(f) Henrique Meirelles

Figura 1. Palavras mais presentes nos tweets dos candidatos

\subsection{Tweets dos candidatos}

A Figura 2 apresenta o número total de tweets coletados por candidato, junto com a quantidade de tweets que o candidato compartilhou de outros usuários do Twitter ao longo de todo o período de coleta de dados. Os três candidatos com maior número de tweets são respectivamente Lula, Henrique Meirelles e João Amoedo. Os candidatos com menor número de tweets são José Maria Eymael, Cabo Daciolo e João Goulart. A figura mostra a quantidade de conteúdos que os candidatos replicam e disseminam. No caso, Lula é o candidato que mais compartilhou tweets de outras pessoas.

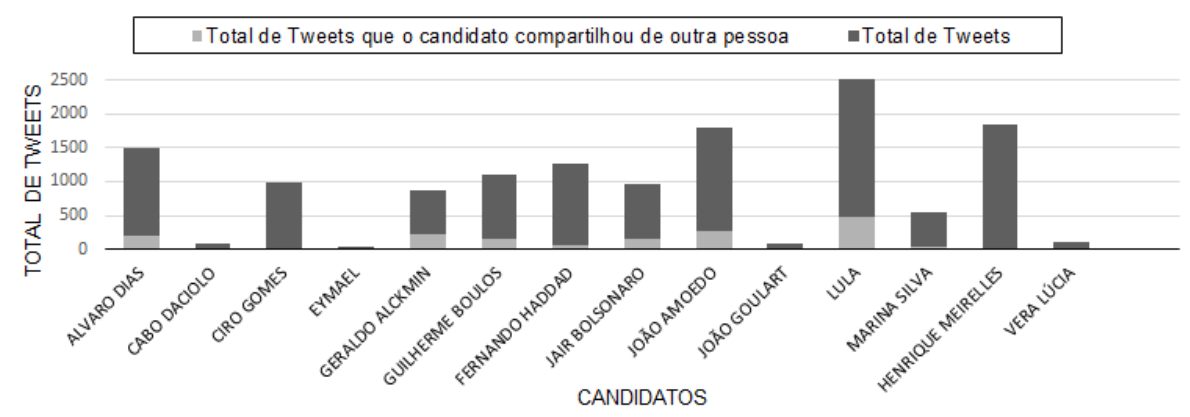

Figura 2. Total de Tweets dos Candidatos

A maior quantidade de tweets compartilhados de outros usuários é realizada por Lula. Porém, o candidato com maior número de compartilhamentos quando comparado ao total de tweets é Geraldo Alckmin, com 33,74\% de retweets. Pode-se perceber também que o candidato José Maria Eymael não retweetou nenhuma publicação de outro usuário do Twitter. 
A Figura 3(a) mostra a soma da quantidade de curtidas de cada tweet. Na figura, os candidatos com maiores valores de curtidas são Jair Bolsonaro, Fernando Haddad e Lula, respectivamente. Os candidatos com menores valores de curtidas são José Maria Eymael, Vera Lúcia e João Goulart.

A Figura 3(b) apresenta o total de retweets que cada tweet obteve. O maior valor de retweets percebe-se nas publicações do candidato Jair Bolsonaro, seguido de Fernando Haddad e Lula. Os menores valores de tweets retweetados são de José Maria Eymael, Cabo Daciolo e João Goulart.

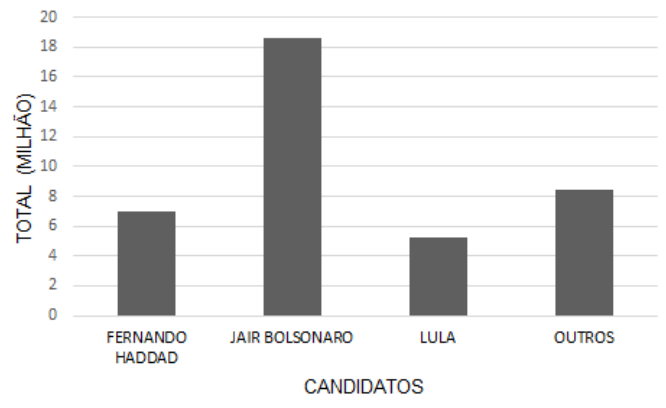

(a) Total de Curtidas

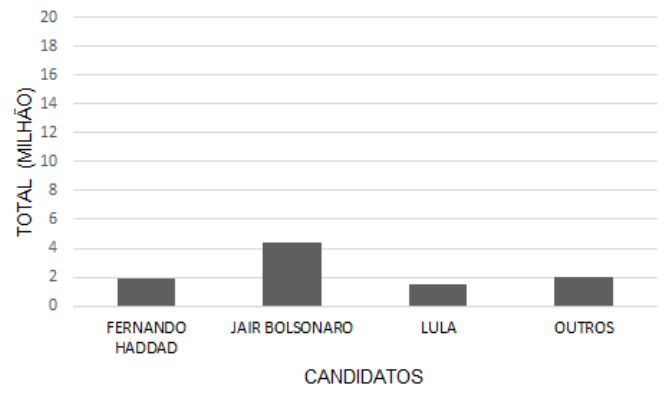

(b) Total de Compartilhamentos

Figura 3. Tweets dos Candidatos

Relacionando-se as Figuras 2, 3(a) e 3(b) pode-se notar que o candidato com menor número de tweets também foi o candidato com menor número de curtidas e menor número de retweets em suas postagens. Ou seja, candidatos com baixa frequência de postagem obtêm menor engajamento dentro da rede social, visto os números de curtidas e retweets de suas publicações. Os candidatos com maiores valores de curtidas também obtiveram maiores valores de retweets.

\subsection{Seguidores dos candidatos}

A Figura 4 mostra a média de seguidores de cada candidato sumarizado de todo o período de coleta. O candidato com maior média de seguidores é a Marina Silva, porém possui o desvio padrão pequeno representando que a dispersão dos números de seus seguidores é pequena. No Twitter a Marina Silva é a candidata com o maior número de seguidores logo no início da campanha e este número tem baixa variação ao longo do tempo. O candidato Jair Bolsonaro apresenta o maior desvio padrão indicando a alta dispersão de número de seguidores durante o período de campanha.

Para analisar a alteração de seguidores ao longo da campanha eleitoral, é apresentado na Figura 5 o percentual de incremento do número de seguidores de cada candidato ao longo do tempo até o final da coleta. Pode-se verificar que o candidato que obteve o maior percentual de incremento foi Cabo Daciolo (Figura 5(b)) passando de 28.697 para 42.888 seguidores, especificamente no dia 30 de Setembro de 2018 que ocorreu o debate presidenciável da emissora Record de televisão ${ }^{9}$. Temos em seguida o candidato João Goulart (Figura 5(c)) passando de 207 para 309 seguidores no dia 30 de Agosto de 2018,

\footnotetext{
${ }^{9}$ Folha De São Paulo. (2018). Saiba Datas E Horários Dos Debates Na TV Antes Do Primeiro Turno Das Eleições 2018. Disponível em: https://goo.gl/cfNcZA . Acesso em: 10 de Setembro de 2018.
} 


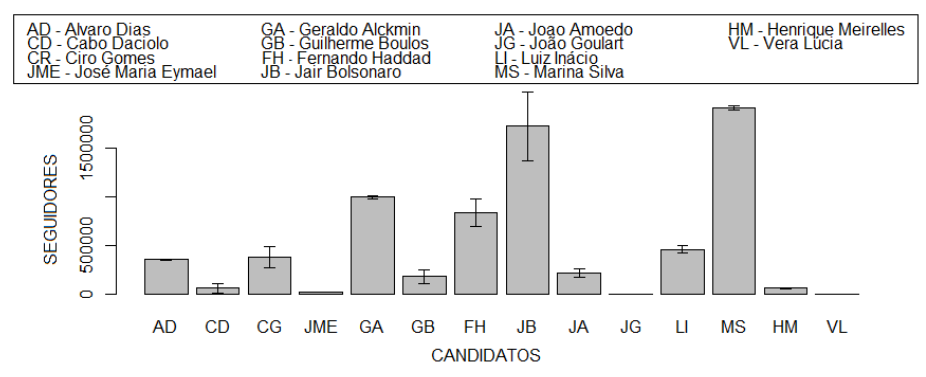

Figura 4. Média e Desvio Padrão de Seguidores dos Candidatos

dia anterior ao início da propaganda eleitoral gratuita no rádio e na televisão. Também foi João Goulart que obteve mais variações de seguidores ao longo da campanha eleitoral.

O candidato que obteve maior decremento foi Álvaro Dias com perda de seguidores (Figura 5(a)) passando de 366.113 para 350.218 seguidores no dia 09 de Novembro de 2018, cuja a data não foi encontrada nenhum evento específico eleitoral na rede de televisão já que o período de campanha eleitoral havia se encerrado.

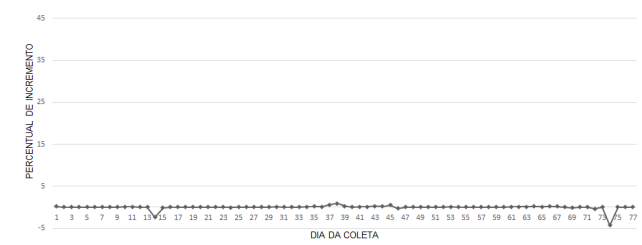

(a) Álvaro Dias

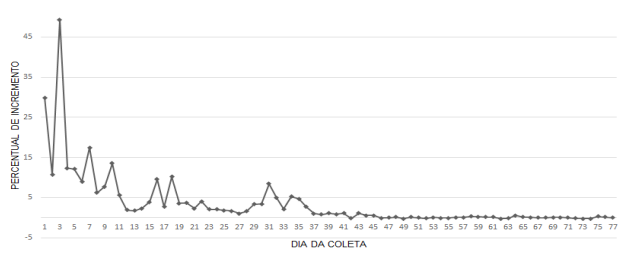

(c) João Goulart

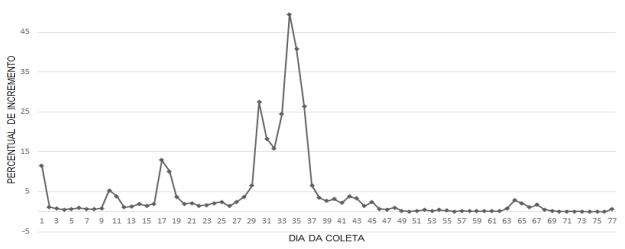

(b) Cabo Daciolo

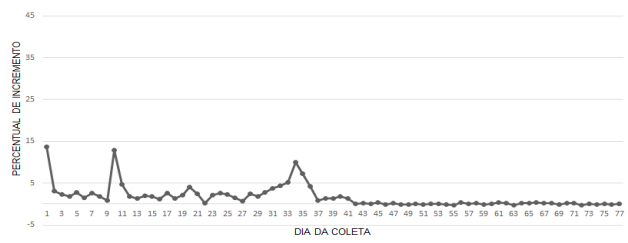

(d) Vera Lúcia

Figura 5. Percentual da variação de Seguidores dos Candidatos - $1^{\circ}$ Turno

A Figura 6 apresenta o percentual de incremento de seguidores dos candidatos calculada também conforme a Equação (1). Foram para o segundo turno os candidatos Jair Bolsonaro e Fernando Haddad. Na figura pode-se perceber que ocorrem dois eventos em que a quantidade de seguidores dos dois candidatos aumentam significativamente: no dia da votação do $1^{\circ}$ Turno, 08 de Outubro de 2018 , e no dia da votação do $2^{\circ}$ Turno, 28 de Outubro de 2018, das eleições presidenciais. Nesta escala de percentual é possível observar também o aumento de seguidores que o candidato Jair Bolsonaro obteve no dia que ocorreu o atentado contra o candidato, 06 de Setembro de 2018, noticiado em todos os canais brasileiros de comunicação [G1 2018]. Jair Bolsonaro no dia que venceu as eleições apresentou maior número de seguidores que seu oponente. 


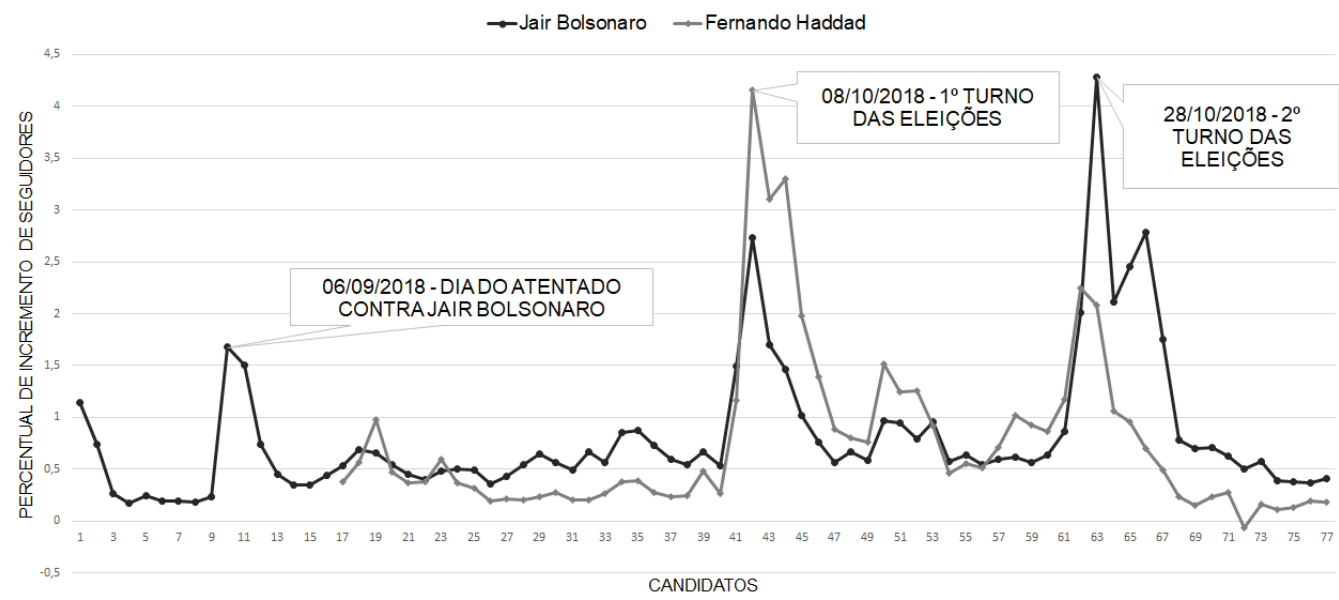

Figura 6. Percentual da variação de Seguidores dos Candidatos - $2^{\circ}$ Turno

\subsection{Citações aos oponentes}

Com o objetivo de avaliar qual a frequência um candidato fala do seu oponente, foi feito um grafo das menções. Esta relação entre os candidatos foi calculada conforme frequência em que um nome de adversário foi mencionado por cada candidato. São apresentados na Figura 7 os resultados obtidos para alguns candidatos.

Cada nó representa um candidato e cada aresta indica a ocorrência de menções ao oponente e a quantidade de vezes ocorridas. O candidato que mais se refere a si mesmo é Henrique Meirelles, 726 vezes. O candidato que menos se refere a si é Vera Lúcia com apenas uma menção a si mesma. Os candidatos José Maria Eymael, João Goulart e Vera Lúcia não foram mencionados por outros candidatos. Jair Bolsonaro é o candidato mais mencionado pelos outros candidatos, ou seja, com maior número de nós ligados a ele em um total de 12 candidatos. Ou seja, o candidato que venceu as eleições foi o mais citado pelos seus oponentes com 601 menções, seguido de Fernando Haddad com 472 menções.

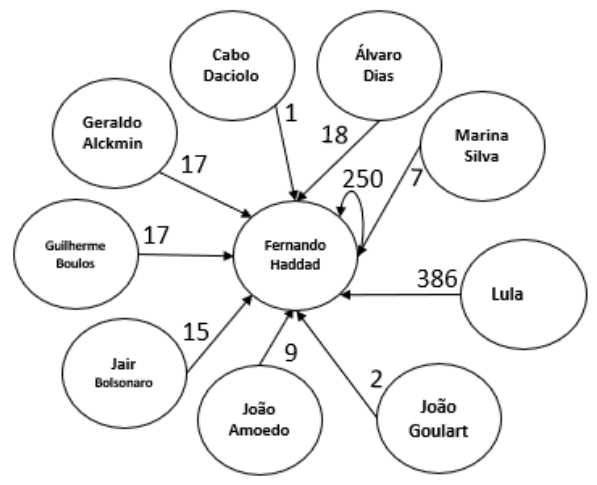

(a) Fernando Haddad

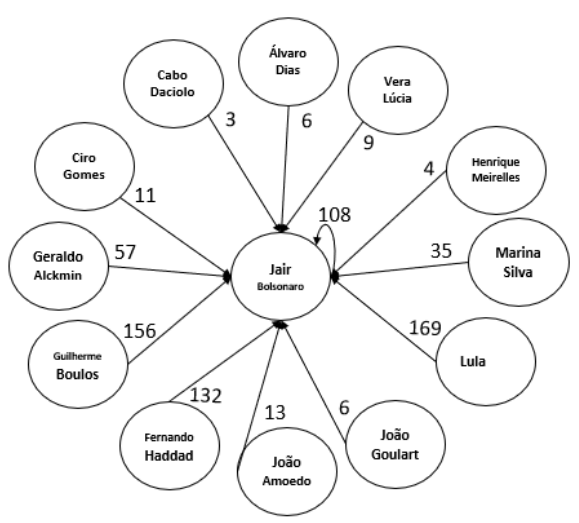

(b) Jair Bolsonaro

Figura 7. Grafo das menções dos candidatos a seus oponentes

\subsection{Termos nos Trending Topics Referentes aos Candidatos}

Para visualizar a ocorrência de termos relacionados aos candidatos, foi analisado todos os termos dos Trending Topics e categorizados por candidato, apresentado na Figura 8. A 
Tabela 1 apresenta exemplos de termos dos Trending Topics e os respectivos candidatos aos quais foram categorizados.

Tabela 1. Exemplos de Termos dos Trending Topics e suas Categorias

\begin{tabular}{|c|c|}
\hline Trending Topic & Candidato Referenciado \\
\hline \#17NOSEGUNDOTURNO & Jair Bolsonaro \\
\hline \#CIRAONAGLOBONEWS & Ciro Gomes \\
\hline \#HADDADE13 & Fernando Haddad \\
\hline
\end{tabular}

O candidato com maior número de menções relacionadas nos Trending Topics é Jair Bolsonaro, seguido de Fernando Haddad e Ciro Gomes. Os candidatos João Goulart, Cabo Daciolo e Henrique Meirelles não tiveram termos relacionados a eles nos trending topics. A maior quantidade de vezes que o candidato Jair Bolsonaro foi mencionado nos trending topics foi no dia anterior ao $2^{\circ}$ Turno das eleições presidenciais. Também relaciona-se que os 3 candidatos mais referenciados nos trending topics foram os 3 candidatos mais bem votados no $1^{\circ}$ turno das eleições.

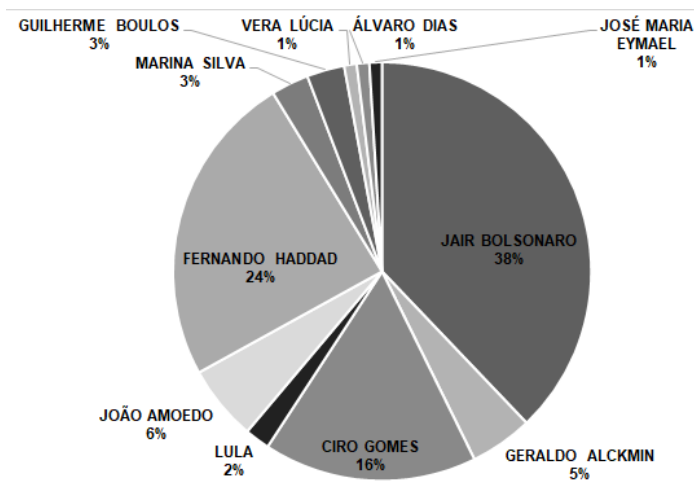

Figura 8. Termos dos Trending Topics Referentes aos Candidatos à Presidência

\section{Conclusões e Trabalhos Futuros}

Nesse artigo foi apresentada uma caracterização das campanhas eleitorais dos candidatos a presidência do Brasil em 2018, na rede social online Twitter, além de incluir um processo de análise de dados que pode ser reproduzido futuramente. Nessa análise foi possível verificar que o candidato vencedor do pleito, Jair Bolsonaro, embora não seja o candidato com maior número de publicações, foi o candidato mais citado pelos opositores, o que mais apareceu referenciado nos trending topics e o candidato com maior total de curtidas e compartilhamentos em suas publicações.

Também pode-se destacar os candidatos Vera Lúcia, José Maria Eymael e João Goulart, que ficaram com as últimas posições nas eleições, não se sobressaíram na rede social Twitter. Foi possível identificar coincidências temporais de acontecimentos noticiados durante as campanhas, exemplificados pelo atentado contra o candidato Jair Bolsonaro e pelos debates presidenciais, que ocasionaram movimentações na rede social.

Como trabalhos futuros pretende-se analisar outras redes sociais nas campanhas eleitorais, comparando o engajamento de usuários e candidatos entre diferentes plataformas. 


\section{Referências}

Attux, R. C. (2017). Predição dos resultados das eleições 2014 para presidente do brasil usando dados do twitter. Trabalho de Conclusão de Curso (Graduação em Sistemas de Informação).

Buchdid, S. B. and Baranauskas, M. C. C. (2012). Ihc em contexto: o que as palavras relevam sobre ela. In Proceedings of the 11th Brazilian Symposium on Human Factors in Computing Systems, pages 199-208. Brazilian Computer Society.

Camargo, A., Silva, R., Amaral, É., Heinen, M., and Pereira, F. (2016). Mineração de dados eleitorais: descoberta de padrões de candidatos a vereador na região da campanha do rio grande do sul. Revista Brasileira de Computação Aplicada, 8(1):64-73.

Cellan-Jones, R. (2016). Como o facebook pode ter ajudado trump a ganhar a eleição. BBC. Disponível em : https://goo.gl/HWaVsv. Acesso em: 30 de Janeiro 2019.

D’Agostino, R. (2018). 13 presidenciáveis registram candidaturas no tse. G1. Disponível em : https://goo.gl/aL5uuZ. Acesso em: 15 de Agosto 2018.

Fourney, A., Racz, M. Z., Ranade, G., Mobius, M., and Horvitz, E. (2017). Geographic and temporal trends in fake news consumption during the 2016 us presidential election. In Proceedings of the 2017 ACM on Conference on Information and Knowledge Management, pages 2071-2074. ACM.

G1 (2018). Jair bolsonaro leva facada durante ato de campanha em juiz de fora. Disponível em : https://goo.gl/uCVZpq. Acesso em 05 de Janeiro de 2019.

Junior, F. M. M., Rodrigues, P. C. D., and Tavares, W. M. (2016). Comportamentos (extremos) dos usuários de redes sociais relativos ao tema política. Múltiplos Olhares em Ciência da Informação-ISSN 2237-6658, 5(2).

Kaniak, T., Paris, L., Gimenes, E., and Rosanne, D. (2018). Pt anuncia candidatura de fernando haddad à presidência no lugar de lula. G1. Disponível em : https://goo.gl/2UJDFG. Acesso em: 11 de Setembro 2018.

Li, H., Dombrowski, L., and Brady, E. (2018). Working toward empowering a community: how immigrant-focused nonprofit organizations use twitter during political conflicts. In Proceedings of the 2018 ACM Conference on Supporting Groupwork, pages 335-346. ACM.

Martins, T. (2018). Dados da internet no brasil em 2018. Marketing Sem Gravata. Disponível em: https://goo.gl/rER3RH. Acesso em: 08 de Agosto 2018.

Miranda Filho, R., Almeida, J. M., and Pappa, G. L. (2014). Pesquisa eleitoral em redes sociais: Inclusão da análise de novas dimensões. In Brazilian Workshop on Social Network Analysis and Mining (BraSNAM).

Recuero, R. and Zago, G. (2016). Em busca das "redes que importam": redes sociais e capital social no twitter. LÍBERO. ISSN impresso: 1517-3283/ISSN online: 2525-3166, (24):81-94.

Vegas, I., Tian, T., and Xiong, W. (2016). Characterizing the 2016 us presidential campaign using twitter data. International Journal of Advanced Computer Science and Applications, 7(10). 